\title{
Quantitative Quasi-Simultaneous and Spatially Resolved Real-time Monitoring of Powder Mixing Processes with a Multiple NIR-Probe Setup
}

\author{
N. BALAK ${ }^{1}$, O. ScheibelHofer ${ }^{1}$, D. M. Koller ${ }^{1}$, J. G. Khinast ${ }^{1,2}$ \\ ${ }_{2}^{1}$ Research Center Pharmaceutical Engineering GmbH, Graz, Austria \\ ${ }^{2}$ Institute for Process and Particle Engineering, Graz University of Technology, Graz, Austria \\ E-mails: nikolaus.balak@rcpe.at (N. Balak), khinast@tugraz.at (J. G. Khinast)
}

Sci Pharm. 2010; 78: 634

doi:10.3797/scipharm.cespt.8.PMS07

Diffuse reflectance near-infrared (NIR) spectroscopy is a non-invasive and widely used technology with the potential for a wide range of applications in the pharmaceutical industry, ranging from raw material identification to final product qualification. Especially powder blending processes, as one of the key unit operations for solid dosage forms can be monitored in real-time for the blend quality and blending time until uniformity. Common NIR systems enable single point measurements at dedicated positions of the blender, based on the assumption, that the sampled position represents the whole blend. This is a problematic task as the mixing characteristics of powders significantly change (e. g., segregation, agglomeration, etc.) with the process parameters.

Here we introduce, a new process analytical technology (PAT) setup for a spatially resolved quasi-simultaneous process monitoring for batch and continuous manufacturing processes. Therefore, an FT-NIR spectrometer was combined with an optical multiplexer, simultaneously switching the excitation and detection path of the bifurcated fibers, which can be connected to a blender via sapphire sampling ports at different positions. Different multi-component powder mixing processes were investigated and quantitatively analyzed with multivariate data analysis (MVDA) using software packages of CAMO and Umetrics, which also support real-time process monitoring.

The new flexible PAT setup presented here, enables non-invasive in-line and real-time monitoring of dynamic processes, especially where spatial deviations can be expected e. g., segregation in solids (i. e., powder blends) and liquids (i. e., sedimentation).

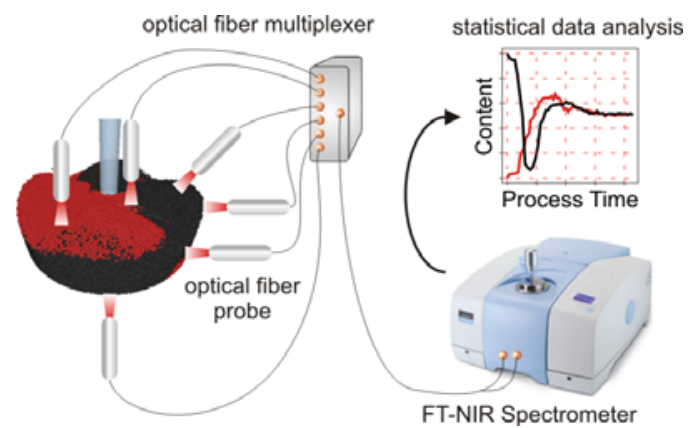

[1] Balak N, Koller DM, Khinast JG. Spatially Resolved Real-time Monitoring of Pharmaceutical Processes with a Multiple NIR-Probe Setup; in preparation. 